\title{
Desarrollo de materiales catalíticos y su aplicación en la revalorización de moléculas plataforma derivadas de biomasa
}

\section{Development of catalytic materials and their application in revalorization of platform molecules derived from biomass}

Presentación: 06/10/2020

\section{Doctorando:}

\section{Georgina Ortenzi}

Centro de Investigación y Tecnología Química (CITeQ), Facultad Regional Córdoba, Universidad Tecnológica Nacional-Argentina gportenzi@gmail.com

\section{Director/a:}

\section{Candelaria Leal Marchena}

\section{Co-director/a:}

\section{Liliana Pierella}

\section{Resumen}

En este trabajo se presentará un resumen del plan de tesis doctoral que consiste en el desarrollo de materiales catalíticos basados en metales de transición y metales nobles impregnados sobre sólidos de elevada área superficial y diferentes propiedades físico-químicas, para ser aplicados en reacciones de valorización de moléculas plataforma derivadas de biomasa. Particularmente, se incorporarán metales de transición ( $\mathrm{Fe}, \mathrm{Co}, \mathrm{Cu}$, entre otros) y metales nobles (Pd, Pt y/o Rh) sobre estructuras organometálicas (MOF-5 y MIL-101), zeolitas comerciales (Y) y alúmina nanotubular $\left(\mathrm{Al}_{2} \mathrm{O}_{3}\right)$. Se evaluarán las propiedades catalíticas de los materiales en reacciones de oxidación de moléculas orgánicas derivadas de la biomasa de la familia de los furanos y compuestos aromáticos (2-furaldehido, 5-hidroximetil-2furaldehido, tolueno), en condiciones suaves o medias que generan un impacto ambiental reducido.

Palabras clave: Materiales catalíticos-Oxidación-Biomasa

\begin{abstract}
This work will summarize the doctoral thesis plan for the development of catalytic materials based on transition metals and noble metals impregnated onto solids with a high surface area and different physical-chemical properties, to be applied in valorization reactions of platform molecules derived from biomass. In particular, transition metals ( $\mathrm{Fe}, \mathrm{Co}$, $\mathrm{Cu}$, among others) and noble metals (Pd, Pt and / or Rh) will be incorporated on organometallic structures (MOF-5 and MIL-101), commercial zeolites $(\mathrm{Y})$ and nanotubular alumina $\left(\mathrm{Al}_{2} \mathrm{O}_{3}\right)$. The catalytic properties of the materials will be evaluated in oxidation reactions of organic molecules derived from the biomass of the furan family and aromatic compounds (2-furaldehyde, 5-hydroxymethyl-2-furaldehyde, toluene), under mild or medium conditions that will generate a reduced environmental impact.
\end{abstract}




\section{Introducción}

En los últimos años, las preocupaciones mundiales del uso de combustibles fósiles para la producción de energía y productos químicos se centran en su disponibilidad limitada y la contaminación causada por su uso, generando la búsqueda de recursos alternativos junto con un control ambiental más severo. Las fuentes de biomasa se presentan como una alternativa más prometedora para el reemplazo de los recursos fósiles, debido a su abundancia en la naturaleza y bajo costo (Albonetti, 2015; Neațu, 2016).

Una alternativa para la valorización de las diferentes biomasas es la pirólisis de las mismas. Este proceso consiste en la degradación térmica en ausencia de oxígeno, dando una fracción sólida (bio-carbón), una líquida (bio-oil) y una gaseosa (Bridgwater, 1996). Las transformaciones catalíticas de biomasa presentan la ventaja de aumentar la eficiencia energética y sustentabilidad de los procesos. En nuestro grupo de trabajo existe una línea de investigación de pirolisis catalítica a partir de las cáscaras de maní para la obtención de bio-oils, ricos en furanos y compuestos aromáticos.

El Departamento de Energía de los Estados Unidos (DOE) publicó un listado de los 10 principales compuestos químicos de base biológica en la cual se mencionan moléculas de la familia de los furanos, como el 5-hidroximetil-2furaldehido (HMF), el 2-furaldehido y el ácido 2,5-furandicarboxílico (FDCA) en el "Top 10 +4" como adiciones a la lista original (Bozell, 2010).

El HMF constituye un precursor para la obtención de derivados combustibles y compuestos químicos claves como precursores poliméricos, agentes antifúngicos, adhesivos y resinas, industria farmacéutica y agroquímica, entre otras (Lilga, 2010, Casanova, 2009, Agirrezabal-Telleria, 2014). Los derivados oxidados de HMF son variados y su naturaleza depende de los catalizadores empleados, así como del medio de reacción, tiempos de contacto y demás condiciones. Como contiene una estructura reactiva que involucra un grupo aldehído, un grupo hidroxilo y un anillo de furano, mediante el empleo de óxidos metálicos como catalizadores se pueden obtener moléculas del corte de combustibles, como 2,5-dimetilfurano (DMF) y 5-etoximetilfurano (EMF) (Hu, 2017), y zeolitas modificadas con metales de transición para la obtención de moléculas con alto valor químico como como ácido levulínico (LA), 2,5-dimetiltetrahidrofurano (DMTHF) y 2-hexanol (HA) (Hu, 2017, Ramli, 2019). El LA puede usarse como aditivos de combustible, material de recubrimiento, solvente, fragancia y saborizante de alimentos, en compuestos farmacéuticos, resinas y biocombustibles. (Ramli, 2019). Se ha reportado también la oxidación de HMF para la obtención de derivados de furanos mediante el empleo de catalizadores heterogéneos soportados basados en $\mathrm{Ru}, \mathrm{V}, \mathrm{Au}$, favoreciendo la obtención de 2,5-diformilfurano (DFF), ácido 5-formil-2-furancarboxílico (FFCA), FDCA, entre otros (Gao, 2017). El FDCA es un componente prometedor ya que se considera como un posible reemplazo del ácido tereftálico, utilizado principalmente como precursor en la producción de polietilenetereftalato (PET) (Bozell, 2010, Martínez-Vargas, 2017).

A partir de la oxidación de 2-furaldehído es posible obtener ácidos orgánicos, como el ácido succínico (AS) y el ácido maleico (AM). El AS es un intermedio de importancia estratégica para la industria química, empleado como precursor de muchos compuestos de importancia industrial (Bozell, 2010). Se ha reportado la oxidación de 2-furaldehído a AS empleando $\mathrm{H}_{2} \mathrm{O}_{2}$ con resinas de intercambio iónico sulfonadas altamente ácidas en medio acuoso y en condiciones suaves de temperatura (Choudhary, 2013). El AM se utiliza en la producción de poliésteres, resinas copolímeros de vinilo, para el recubrimiento de superficie y en aceites (Alba-Rubio, 2017). Se han empleado materiales mesoporosos empleados como soportes de catalizadores para la obtención de AM a partir de la oxidación de 2-furaldehído (Rezaei, 2019).

La oxidación selectiva de compuestos aromáticos permite obtener un amplio rango de moléculas de valor agregado e intermediarias de síntesis para la química fina. El tolueno es un compuesto aromático que proporciona tres enlaces $\mathrm{C}$ - $\mathrm{H}$ y puede oxidarse a benzaldehído (BZ), ácido benzoico (AB), alcohol bencílico (B-ol), entre otros. El BZ es un producto de gran interés industrial debido a su aplicación como solventes, perfumes, colorantes y plastificantes (Xia, 2016). El AB presenta gran demanda en la industria actual debido a su aplicación en medicamentos, plastificantes, colorantes, conservantes, entre otros (Gizli, 2008). Se reportó el uso de zeolitas funcionalizadas con Cu en la oxidación selectiva de tolueno para la obtención de AB (Viswanadham, 2017). 
Las estructuras organometálicas (MOF) representan una clase de materiales porosos. Se pueden distinguir dos componentes en los MOF: unidades de construcción secundarias (grupos o iones metálicos) y moléculas orgánicas que unen las primeras para dar estructuras porosas básicamente periódicas. La elección de estos componentes permite variar sus propiedades, lo que abre nuevas vías de producción de materiales con propiedades fisicoquímicas adaptadas a requerimientos específicos. La capacidad de incorporar grupos funcionales en estos materiales porosos los convierte en excelentes candidatos como catalizadores heterogéneos (Butova, 2016, Ma, 2009, Qiu, 2009). La modificación permite crear nuevos sitios catalíticos o activar los existentes. Los nuevos sitios catalíticos se pueden obtener mediante la introducción de una nanopartícula de metales de transición o metales nobles en los poros y superficie, cambiando el entorno del ligando de los sitios metálicos o modificando los enlaces orgánicos. Las nanopartículas encapsuladas en los MOF exhiben estabilidad, alta dispersión, tamaño y evitan la aglomeración de las partículas (Cohen, 2012, Klet, 2016).

La alúmina $\left(\mathrm{Al}_{2} \mathrm{O}_{3}\right)$ es uno de los óxidos más importantes debido a que presenta buena estabilidad térmica, superficie específica elevada, propiedades ácido-base modulables, bajo costo y facilidad de preparación. Estas características hacen que este material sea atractivo para aplicaciones de absorbente, catalizador y soporte de catalizadores heterogéneos. Dentro de la amplia gama de nanoestructuras, la tubular es una de las de mayor interés debido a las múltiples y variadas aplicaciones. Se ha reportado la síntesis nanotubos $\gamma-\mathrm{Al}_{2} \mathrm{O}_{3}$ los cuales presentan microporosidad y mesoporosidad. La existencia de microporos en los nanotubos sugiere la formación de cavidades dentro de la pared que mejoran la difusión y poseen un área superficial efectiva relativamente grande (Qu, 2005, Lu, 2009, Zhao, 2015).

Las zeolitas consisten en una red tridimensional de tetraedros metal-oxígeno, donde los sitios activos son parte de la estructura, y resultan del desbalance que surge de la coordinación tetraédrica del metal con los tetraedros de silicio. Particularmente resultan interesantes, las zeolitas de poro grande $Y$ con área superficial entre $500-600 \mathrm{~m}^{2} / \mathrm{g}$. Esta conjunción de red eléctricamente cargada, elevada área superficial, conformación estructural de poros y canales bien definidos y estables, las hacen altamente atractivas como materiales catalíticos (Xu, 2006) o bien, para su empleo como soportes de especies activas (Saux, 2016, Gu, 2015). Se ha reportado el empleo de zeolitas como catalizadores para la oxidación de numerosos derivados de biomasa (Perego, 2011). Además, la actividad catalítica y selectividad (con rendimientos del 99\%) de materiales zeolíticos y mesoporosos fue estudiada en la conversión de HMF (Casanova, 2010).

Con la idea de avanzar en el estudio de valorización de la biomasa, nos proponemos en el presente plan de trabajo de tesis doctoral avanzar en esta última etapa desarrollando materiales catalíticos para la valorización de moléculas de la familia de los furanos y compuestos aromáticos.

\section{Desarrollo}

En primera instancia se sintetizarán los diferentes materiales catalíticos descriptos anteriormente: las estructuras organometálicas (MOF-5 y MIL-101) por síntesis solvotermal. Para MOF-5, la síntesis consiste en la adición directa de trietilamina a una solución de $\mathrm{N}, \mathrm{N}^{\prime}$-dimetilformamida y clorobenceno conteniendo $\mathrm{Zn}\left(\mathrm{NO}_{3}\right)_{2} \cdot 6 \mathrm{H}_{2} \mathrm{O}$ y ácido 4bencenodicarboxílico. Se añade una pequeña cantidad de $\mathrm{H}_{2} \mathrm{O}_{2}$ para facilitar la formación de $\mathrm{O}^{2-}$ deseables en el centro de la unidad secundaria. Los MIL-101 se sintetizan a partir de $\mathrm{CrCl}_{3} .6 \mathrm{H}_{2} \mathrm{O}$, ácido tereftálico y agua desionizada colocado en reactores con presión autógena revestidos de teflón a $210^{\circ} \mathrm{C}$. Los nanotubos de $\mathrm{Al}_{2} \mathrm{O}_{3}$ se sintetizarán empleando una ruta hidrotermal. Se empleará como agente director de estructura dodecilsulfonato sódico disuelto en agua destilada; nitrato de aluminio hidratado como precursor y amoníaco como regulador de $\mathrm{pH}$. La zeolita Y se obtendrá de manera comercial.

Se incorporarán diferentes funciones activas a los soportes sólidos catalíticos, metales de transición ( $\mathrm{Fe}$, Co, Cu, entre otros) y metales nobles ( $\mathrm{Pt}, \mathrm{Pd}$ y/o $\mathrm{Rh}$ ). El dopaje de las mismas se realizará por impregnación vía húmeda a partir de sus sales correspondientes (acetatos, nitratos, cloruros) con la cantidad de metal que se desea incorporar. Luego, a los efectos de determinar cristalinidad, estructura, tamaño y morfología de los cristales, cantidad y tipo de sitios activos, contenido metálico, etc., se realizará la caracterización de los materiales por las siguientes técnicas: Espectroscopia Fotoelectrónica de rayos X (XPS); Análisis Térmico Diferencial (DTA) y Termogravimetría (TGA); Superficie específica por adsorción de nitrógeno a 77K; Microscopía electrónica de barrido (SEM); Desorción térmica de $\mathrm{O}_{2}$ (TPO); Reducción Térmica Programada (TPR); Espectroscopia Infrarroja con Transformada de Fourier (FTIR) por adsorción de moléculas 
sonda; Difracción de Rayos X (XRD); Espectroscopia Infrarroja con Transformada de Fourier (FTIR); Espectroscopia UltraVioleta-Reflectancia Difusa (UV-Vis-DRS), entre otras.

La evaluación catalítica de estos materiales se efectuará en un reactor de vidrio Pirex, sumergido en un baño termostatizado operando a presión atmosférica, equipado con un condensador a reflujo, termómetro y agitación magnética manteniendo el catalizador en suspensión. También se prevé utilizar un reactor de alta presión tipo PARR. Se evaluarán variables como tiempos de reacción, temperatura, influencia de disolventes, masa de catalizador, relación sustrato/oxidante, reutilización y reúsos de los catalizadores, a los fines de determinar las condiciones óptimas de trabajo, ajustar el sistema, los reactivos y los materiales. Los productos de reacción se identificarán y cuantificarán por cromatografía líquida de alta resolución (HPLC) empleando un cromatógrafo Perkin Elmer Serie 200 con detector UVVis, y/o un cromatógrafo en fase gaseosa (CG) Perkin Elmer Clarus 500, equipado con una columna capilar y un detector FID; y complementando las mediciones con espectrometría de masas empleando un equipo GC Masas (Shimadzu QP 5050 GC-17 A), con una columna capilar.

\section{Conclusiones}

De lo anteriormente expuesto, se plantea la valorización de moléculas obtenidas de la pirólisis de biomasa mediante oxidación catalítica para la obtención de compuestos de interés de química fina e intermediarios de síntesis. Se sintetizarán y emplearán catalizadores heterogéneos modificados con metales de transición y metales nobles a los fines de evaluar el efecto de las diferentes estructuras tanto en la incorporación de las especies activas como en la actividad catalítica; planteando que el uso de éstos mejorará las condiciones de reacción, reduciendo la distribución de productos, permitiendo alcanzar mayor selectividad hacia aquellos de particular interés, además de reducir los tiempos de reacción y las temperaturas del proceso.

\section{Referencias}

Agirrezabal-Telleria, I., Gandarias, I., \& Arias, P. L. (2014). Heterogeneous acid-catalysts for the production of furanderived compounds (furfural and hydroxymethylfurfural) from renewable carbohydrates: a review. Catalysis Today, 234, 42-58. doi.org/10.1016/j.cattod.2013.11.027

Alba-Rubio, A. C., Fierro, J. L. G., León-Reina, L., Mariscal, R., Dumesic, J. A., \& Granados, M. L. (2017). Oxidation of furfural in aqueous $\mathrm{H} 2 \mathrm{O} 2$ catalysed by titanium silicalite: deactivation processes and role of extraframework Ti oxides. Applied Catalysis B: Environmental, 202, 269-280.. doi.org/10.1016/j.apcatb.2016.09.025

Albonetti, S., Lolli, A., Morandi, V., Migliori, A., Lucarelli, C., \& Cavani, F. (2015). Conversion of 5-hydroxymethylfurfural to 2, 5-furandicarboxylic acid over Au-based catalysts: Optimization of active phase and metal-support interaction. Applied Catalysis B: Environmental, 163, 520-530. doi.org/10.1016/j.apcatb.2014.08.026

Bozell, J. J., \& Petersen, G. R. (2010). Technology development for the production of biobased products from biorefinery carbohydrates-the US Department of Energy’s “Top 10” revisited. Green Chemistry, 12(4), 539-554.

doi.org/10.1039/b922014c

Bridgwater, A. V. (1996). Production of high-grade fuels and chemicals from catalytic pyrolysis of biomass. Catalysis today, 29(1-4), 285-295. doi.org/10.1016/0920-5861 (95)00294-4

Butova, V. V. E., Soldatov, M. A., Guda, A. A., Lomachenko, K. A., \& Lamberti, C. (2016). Metal-organic frameworks: structure, properties, methods of synthesis and characterization. Russian Chemical Reviews, 85(3), 280. doi.org/10.1070/RCR4554.

Casanova, O., Iborra, S., \& Corma, A. (2009). Biomass into chemicals: One pot-base free oxidative esterification of 5hydroxymethyl-2-furfural into 2, 5-dimethylfuroate with gold on nanoparticulated ceria. Journal of Catalysis, 265(1), 109-116. doi.org/10.1016/j.jcat.2009.04.019

Casanova, O., Iborra, S., \& Corma, A. (2010). Chemicals from biomass: Etherification of 5-hydroxymethyl-2-furfural (HMF) into 5, 5' (oxy-bis (methylene)) bis-2-furfural (OBMF) with solid catalysts. Journal of Catalysis, 275(2), 236-242. doi.org/10.1016/j.jcat.2010.08.002 
Choudhary, H., Nishimura, S., \& Ebitani, K. (2013). Metal-free oxidative synthesis of succinic acid from biomass-derived furan compounds using a solid acid catalyst with hydrogen peroxide. Applied Catalysis A: General, 458, 55-62. doi.org/10.1016/j.apcata.2013.03.033

Cohen, S. M. (2012). Postsynthetic methods for the functionalization of metal-organic frameworks. Chemical reviews, 112(2), 970-1000. doi.org/10.1021/cr200179u

Gao, T., Gao, T., Fang, W., \& Cao, Q. (2017). Base-free aerobic oxidation of 5-hydroxymethylfurfural to 2, 5furandicarboxylic acid in water by hydrotalcite-activated carbon composite supported gold catalyst. Molecular Catalysis, 439, 171-179. doi.org/10.1016/j.mcat.2017.06.034

Gizli, A., Aytimur, G., Alpay, E., \& Atalay, S. (2008). Catalytic liquid phase oxidation of toluene to benzoic acid. Chemical Engineering \& Technology: Industrial Chemistry-Plant Equipment-Process Engineering-Biotechnology, 31(3), 409-416. doi.org/10.1002/ceat.200700140

Gu, X., Lu, H., Kan, C., \& Yao, J. (2015). One-pot hydrothermal synthesis of zeolite/sodium tantalate composite and its photodegradation of methyl orange. Materials Research Bulletin, 68, 185-188.

doi.org/10.1016/j.materresbull.2015.03.046

Hu, L., Lin, L., Wu, Z., Zhou, S., \& Liu, S. (2017). Recent advances in catalytic transformation of biomass-derived 5hydroxymethylfurfural into the innovative fuels and chemicals. Renewable and Sustainable Energy Reviews, 74, 230257. doi.org/10.1016/j.rser.2017.02.042

Klet, R. C., Wang, T. C., Fernandez, L. E., Truhlar, D. G., Hupp, J. T., \& Farha, O. K. (2016). Synthetic Access to Atomically Dispersed Metals in Metal-Organic Frameworks via a Combined Atomic-Layer-Deposition-in-MOF and Metal-Exchange Approach. Chemistry of Materials, 28(4), 1213-1219. doi.org/10.1021/acs.chemmater.5b04887

Lilga, M. A., Hallen, R. T., \& Gray, M. (2010). Production of oxidized derivatives of 5-hydroxymethylfurfural (HMF). Topics in Catalysis, 53(15-18), 1264-1269. doi.org/10.1007/s11244-010-9579-4.

Lu, C. L., Lv, J. G., Xu, L., Guo, X. F., Hou, W. H., Hu, Y., \& Huang, H. (2009). Crystalline nanotubes of $\gamma$-AlOOH and $\gamma$-Al203: hydrothermal synthesis, formation mechanism and catalytic performance. Nanotechnology, 20(21), 215604.

doi.org/10.1088/0957-4484/20/21/215604

Ma, L., Abney, C., \& Lin, W. (2009). Enantioselective catalysis with homochiral metal-organic frameworks. Chemical Society Reviews, 38(5), 1248-1256. doi.org/10.1039/b807083k

Martínez-Vargas, D. X., De La Rosa, J. R., Sandoval-Rangel, L., Guzmán-Mar, J. L., Garza-Navarro, M. A., Lucio-Ortiz, C. J., \& De Haro-Del Río, D. A. (2017). 5-Hydroxymethylfurfural catalytic oxidation under mild conditions by Co (II), Fe (III) and $\mathrm{Cu}$ (II) Salen complexes supported on SBA-15: Synthesis, characterization and activity. Applied Catalysis A: General, 547, 132-145. doi.org/10.1016/j.apcata.2017.08.035

Neațu, F., Marin, R. S., Florea, M., Petrea, N., Pavel, O. D., \& Pârvulescu, V. I. (2016). Selective oxidation of 5hydroxymethyl furfural over non-precious metal heterogeneous catalysts.Applied catalysis b: environmental, 180, 751757. doi.org/10.1016/j.apcatb.2015.07.043

Perego, C., \& Bosetti, A. (2011). Biomass to fuels: The role of zeolite and mesoporous materials. Microporous and Mesoporous Materials, 144(1-3), 28-39. doi.org/10.1016/j.micromeso.2010.11.034

Qiu, S., \& Zhu, G. (2009). Molecular engineering for synthesizing novel structures of metal-organic frameworks with multifunctional properties. Coordination Chemistry Reviews, 253(23-24), 2891-2911.

doi.org/10.1016/j.ccr.2009.07.020

Qu, L., He, C., Yang, Y., He, Y., \& Liu, Z. (2005). Hydrothermal synthesis of alumina nanotubes templated by anionic surfactant. Materials Letters, 59(29-30), 4034-4037. doi.org/10.1016/j.matlet.2005.07.059

Ramli, N. A. S., \& Amin, N. A. S. (2016). Kinetic study of glucose conversion to levulinic acid over Fe/HY zeolite catalyst. Chemical Engineering Journal, 283, 150-159.doi.org/10.1016/j.cej.2015.07.044 
Rezaei, M., Chermahini, A. N., Dabbagh, H. A., Saraji, M., \& Shahvar, A. (2019). Furfural oxidation to maleic acid with H2O2 by using vanadyl pyrophosphate and zirconium pyrophosphate supported on well-ordered mesoporous KIT-6. Journal of Environmental Chemical Engineering, 7(1), 102855. doi.org/10.1016/j.jece.2018.102855

Saux, C., Leal Marchena, C., Pizzio, L. R., \& Pierella, L. B. (2016). Dodecatungstocobaltate supported over ZSM-5 zeolite as novel solid catalyst in selective sulfide oxidation. Journal of Porous Materials, 23(4), 947-956. doi.org/10.1007/s10934016-0152-9

Viswanadham, N., Saxena, S. K., \& Ala'a, H. (2017). Cu functionalized nano crystalline ZSM-5 as efficient catalyst for selective oxidation of toluene. Materials Today Chemistry, 3, 37-48. doi.org/10.1016/j.mtchem.2017.01.001

Xia, H., Liu, Z., Xu, Y., Zuo, J., \& Qin, Z. (2016). Highly efficient V-Mo-Fe-O catalysts for selective oxidation of toluene to benzaldehyde. Catalysis Communications, 86, 72-76. doi.org/10.1016/j.catcom.2016.08.008

Xu, B., Rotunno, F., Bordiga, S., Prins, R., \& van Bokhoven, J. A. (2006). Reversibility of structural collapse in zeolite Y: Alkane cracking and characterization. Journal of catalysis, 241(1), 66-73. doi.org/10.1016/j.jcat.2006.04.009

Zhao, G., Xia, L., Zhong, B., Song, L., \& Wen, G. (2015). Large-scale fast synthesis of single-crystalline alpha-alumina nanotubes. Ceramics International, 41(2), 2590-2593. doi.org/10.1016/j.ceramint.2014.08.110 